\title{
Epithelioid trophoblastic tumor found on hysteroscopy
}

\author{
Di Zhang ${ }^{1}$, Wenchao Sun ${ }^{2}$, Dingheng Li $^{2}$, Zhifen Zhang ${ }^{2}$ \\ ${ }^{1}$ Department of Reproductive Medicine, Shanghai Tenth People's Hospital, Shanghai 200072, China; ${ }^{2}$ Department of Gynecology, Hangzhou \\ Women's Hospital, Hangzhou 310000, China \\ Correspondence to: Zhifen Zhang. Hangzhou Women’s Hospital, 369 Kunpeng Road, Hangzhou 310000, China. Email: zhangzf@zju.edu.cn.
}

\begin{abstract}
A 36-year-old woman presented with a history of prolonged menstrual period and increased menstrual volume of 4 months. Ultrasonography showed inhomogeneous echo measuring $2.5 \times 1.9 \times 2.2 \mathrm{~cm}^{3}$ in uterine cavity, and it can be seen that the blood flow signal enters the uterine posterior wall. Trophoblastic disease was not ruled out. But the serum $\beta$-human chorionic gonadotropin (hCG) was $<0.3 \mathrm{mIU} / \mathrm{mL}$. In order to confirm the diagnosis, the patient was planned to undergo hysteroscopy. Hysteroscopy is an ideal solution for early diagnosis. However, the drawback of hysteroscopy is that only local lesions can be removed. If the infiltration degree is deep, a second hysterectomy is required. Our authors present the first case of epithelioid trophoblastic tumor (ETT) under hysteroscopy. After neoplasm partial resection, histopathological examination revealed ETT. The patient underwent hysterectomy to prevent recurrence.
\end{abstract}

Keywords: Epithelioid trophoblastic tumor (ETT); hysteroscopy; case report

Submitted Jul 13, 2019. Accepted for publication Nov 22, 2019.

doi: $10.21037 /$ tcr.2019.12.24

View this article at: http://dx.doi.org/10.21037/tcr.2019.12.24

Epithelioid trophoblastic tumor (ETT) is a rare kind of gestational trophoblastic tumor (GTN), which was previously termed atypical choriocarcinoma $(1,2)$. Its biological behavior is different from the general GTN, but similar to placental site trophoblastic tumor (PSTT). PSTT and ETT are rare types of GTN both arise from intermediate trophoblast (3). Because of the cell of origin is different from other forms of GTN, not only clinical presentation, but also tumor marker profile, and treatment paradigm for PSTT and ETT are quite different. Due to its diverse performance, ETT is often misdiagnosed as choriocarcinoma, uterine fibroids, ectopic pregnancy or cervical cancer before surgery. Most ETT cases are incidentally diagnosed postoperatively. Here we present the first case of ETT under hysteroscopy.

A 36-year-old woman presented with a history of prolonged menstrual period and increased menstrual volume of 4 months. There was no disease-related interventions and treatments before coming to our hospital. The patient has a two years old daughter after cesarean section. She breastfed for 18 months and started to have period 7 months after giving birth. She denied cancer, genetic disease and family history in three generations of two families. Her family relations are harmonious. Gynecological examination results were normal—cervix: normal size, smooth surface, no contact bleeding, no lifting pain; uterus: anterior position, regular size, regular shape, without mass, no tenderness, good mobility. Ultrasonography showed inhomogeneous echo measuring $2.5 \times 1.9 \times 2.2 \mathrm{~cm}^{3}$ in uterine cavity, and it can be seen that the blood flow signal enters the uterine posterior wall. Trophoblastic disease was not ruled out. But the serum $\beta$-human chorionic gonadotropin (hCG) was $<0.3$ $\mathrm{mIU} / \mathrm{mL}$. In order to confirm the diagnosis, the patient was planned to undergo hysteroscopy.

Hysteroscopy revealed a neoplasm sticking out of the cavity that measuring $2 \times 2 \times 1.5 \mathrm{~cm}^{3}$ in size in the left side of uterine cavity with thickened endometrium (Figure 1). The neoplasm was friable and the base part of which was broad. Surface vessels of the neoplasm were rich and tortuous (Figure 2). The patient underwent hysteroscopic neoplasm resection. After neoplasm partial resection, hysteroscopy revealed dense and visible openings of endometrium gland (Figure 3). Histopathological examination revealed ETT. ETT cells arranged in the nest bulk, invading the muscle layer (Figure 4). The cells are consisted of relatively uniform nuclei and clear cytoplasm surrounded by a well-defined cell membrane (Figure 5). After the diagnosis, the patient agreed to undergo hysterectomy, and the second histopathological examination did not find ETT lesions throughout the uterus, 


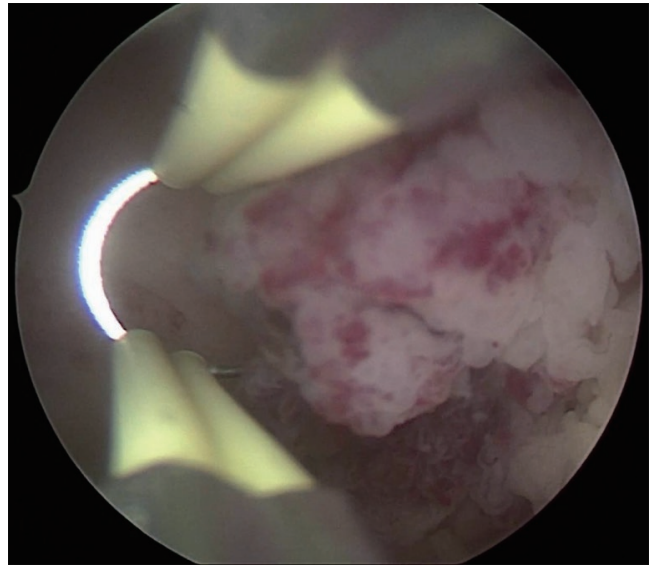

Figure 1 A neoplasm sticking out of the cavity that measuring $2 \times 2 \times 1.5 \mathrm{~cm}^{3}$ in size in the left side of uterine cavity.

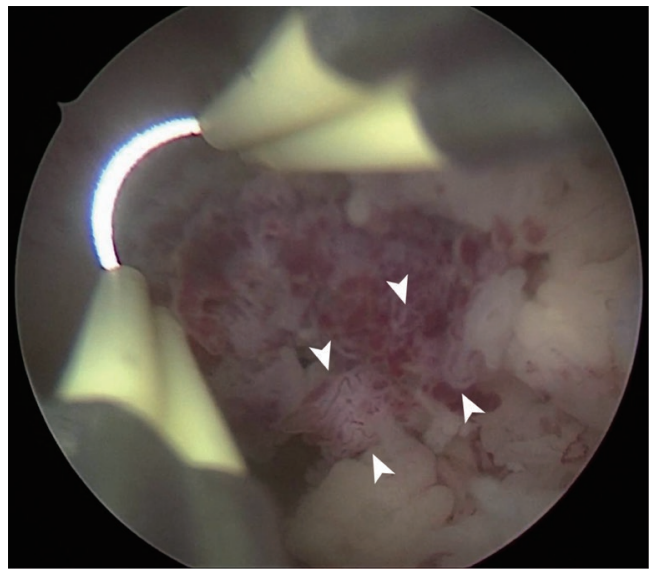

Figure 2 Surface vessels (arrow heads) of the neoplasm were rich and tortuous.

therefore, there is no chemotherapy. The patient had no signs of recurrence after one year of follow-up, and we would continue to follow up. She is satisfied with our treatment. Figure 6 shows the timeline of the patient disease treatment.

Most of ETT patients present at reproductive age, ranging from 15 to 48 years (average 36.1 years) $(4,5)$. They usually have a history of previous pregnancy, accompanied with irregular vaginal bleeding. These clinical features were consistent with our case. But they usually have a slightly to moderately elevated HCG (6), in this case, HCG was negative. Therefore, in the case that only ultrasound is available as a positive auxiliary examination, it is difficult to distinguish between ETT and endometrial polyp or other endometrial disease at early stage of the disease. In this case, Figure 3

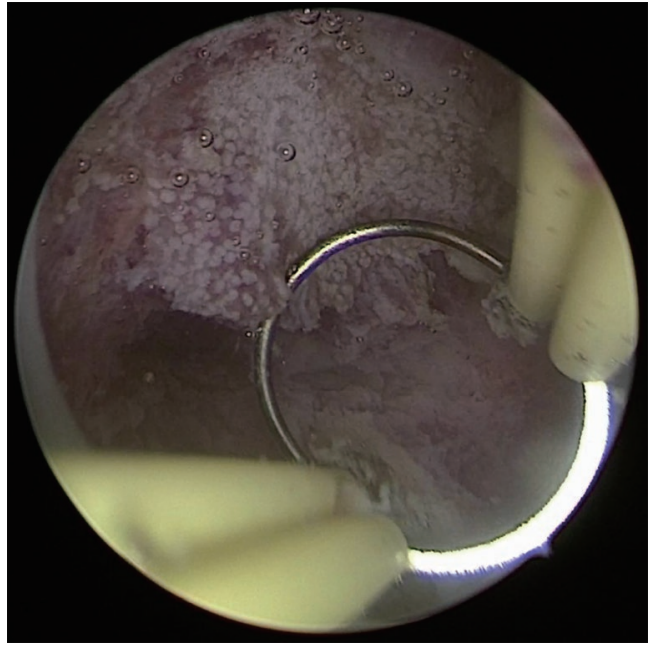

Figure 3 After neoplasm partial resection, hysteroscopy revealed dense and visible openings of endometrium gland.

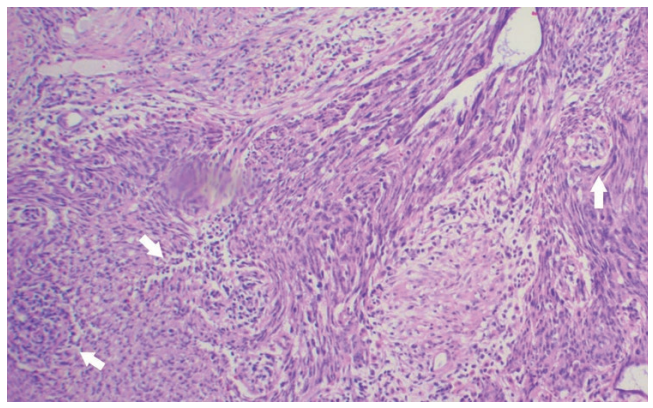

Figure 4 ETT cells arranged in the nest bulk, invading the muscle layer (arrows). Tissues were stained with HE. Image was taken at 200× magnification. ETT, epithelioid trophoblastic tumor.

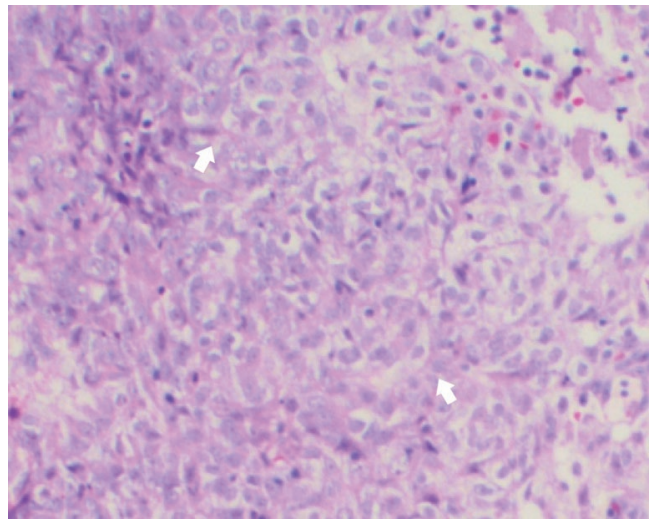

Figure 5 ETT cells have neclei, clear cell boundaries, and transparent cytoplasm (arrows). Tissues were stained with HE. Image was taken at 200× magnification. ETT, epithelioid trophoblastic tumor. 


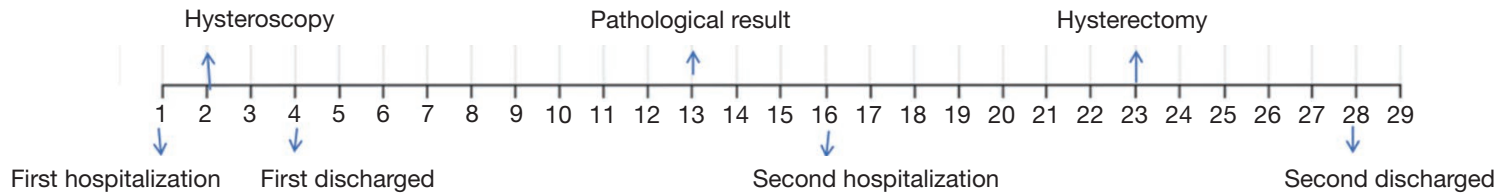

Figure 6 Timeline of disease treatment process.

shows the dense and visible openings of endometrium gland of neoplasm, it may be a unique manifestation of this disease before histopathological examination.

Currently, there are no long-term follow-up materials and its biological behavior is unclear. It generally shows benign behavior and is deemed a disease with low malignant potential. Due to its rarity, experience with ETT is limited. So far, at most 110 cases of this kind of rare tumor have been reported, with clinical feature or pathological characteristics being focused mostly. Jiang et al. have reported laparoscopy is a safe and efficient method for selected patients (7). But for patients with early disease, laparoscopic exploration is not the preferred method because of the greater surgical trauma. Hysteroscopy is an ideal solution for early diagnosis and treatment of disease. However, the drawback of hysteroscopy is that only local lesions can be removed. If the infiltration degree is deep, a second hysterectomy is required. So far tumor morphology under hysteroscopy has not yet been described in detail. This case highlights high definition pictures of ETT under hysteroscopy so that doctors can have a morphological understanding of ETT.

\section{Acknowledgments}

Funding: None.

\section{Footnote}

Conflicts of Interest: All authors have completed the ICMJE uniform disclosure form (available at http://dx.doi. org/10.21037/tcr.2019.12.24). The authors have no conflicts of interest to declare.

Ethical Statement: The authors are accountable for all aspects of the work in ensuring that questions related to the accuracy or integrity of any part of the work are appropriately investigated and resolved. All procedures performed in studies involving human participants were in accordance with the Helsinki Declaration (as revised in 2013). Written informed consent was obtained from the patient for publication of this manuscript and any accompanying images.
Open Access Statement: This is an Open Access article distributed in accordance with the Creative Commons Attribution-NonCommercial-NoDerivs 4.0 International License (CC BY-NC-ND 4.0), which permits the noncommercial replication and distribution of the article with the strict proviso that no changes or edits are made and the original work is properly cited (including links to both the formal publication through the relevant DOI and the license). See: https://creativecommons.org/licenses/by-nc-nd/4.0/.

\section{References}

1. Lurain JR. Gestational trophoblastic disease I: epidemiology, pathology, clinical presentation and diagnosis of gestational trophoblastic disease, and management of hydatidiform mole. Am J Obstet Gynecol 2010;203:531-9.

2. Lurain JR. Gestational trophoblastic disease II: classification and management of gestational trophoblastic neoplasia. Am J Obstet Gynecol 2011;204:11-8.

3. Horowitz NS, Goldstein DP, Berkowitz RS. Placental site trophoblastic tumors and epithelioid trophoblastic tumors: Biology, natural history, and treatment modalities. Gynecol Oncol 2017;144:208-14.

4. Coulson LE, Kong CS, Zaloudek C. Epithelioid trophoblastic tumor of the uterus in a postmenopausal woman: a case report and review of the literature. Am J Surg Pathol 2000;24:1558-62.

5. Olson MT, Gocke CD, Giuntoli RL, 2nd, et al. Evolution of a trophoblastic tumor from an endometrioid carcinoma-a morphological and molecular analysis. Int J Gynecol Pathol 2011;30:117-20.

6. Zhang $\mathrm{X}, \mathrm{Lu} \mathrm{W}, \mathrm{Lu}$ B. Epithelioid trophoblastic tumor: an outcome-based literature review of 78 reported cases. Int J Gynecol Cancer 2013;23:1334-8.

7. Jiang F, Xiang Y, Guo LN. Laparoscopic diagnosis and treatment of an isolated epithelioid trophoblastic tumor in recto-uterine pouch. J Obstet Gynaecol Res 2018;44:960-5.

Cite this article as: Zhang D, Sun W, Li D, Zhang Z. Epithelioid trophoblastic tumor found on hysteroscopy. Transl Cancer Res 2020;9(3):2037-2039. doi: 10.21037/tcr.2019.12.24 